\title{
Inflation, baryogenesis, and gravitino dark matter at ultralow reheat temperatures
}

\author{
Kazunori Kohri, ${ }^{1}$ Anupam Mazumdar, ${ }^{1,2}$ and Narendra Sahu ${ }^{1}$ \\ ${ }^{1}$ Physics Department, Lancaster University, Lancaster, LA1 4YB, United Kingdom \\ ${ }^{2}$ Niels Bohr Institute, Blegdamsvej-17, Copenhagen, DK-2100, Denmark
}

(Received 1 June 2009; published 5 November 2009)

\begin{abstract}
It is quite possible that the reheat temperature of the Universe is extremely low close to the scale of big bang nucleosynthesis, i.e. $T_{R} \sim 1-10 \mathrm{MeV}$. At such low reheat temperatures generating matter, antimatter asymmetry and synthesizing dark matter particles are challenging issues which need to be addressed within a framework of beyond the standard model physics. In this paper we point out that a successful cosmology can emerge naturally provided the $R$-parity violating interactions are responsible for the excess in baryons over antibaryons and at the same time they can explain the longevity of dark matter with the right abundance.
\end{abstract}

DOI: 10.1103/PhysRevD.80.103504

PACS numbers: $98.80 . \mathrm{Cq}, 95.35 .+\mathrm{d}$

Our Universe might have gone through multiple phases of inflation; see, for an example, [1]. It is paramount that the last phase of inflation must provide sufficient e-foldings of inflation to explain the large scale structure of the Universe besides providing the seed perturbations for the temperature anisotropy for the cosmic microwave background $(\mathrm{CMB})$ radiation [2]. It is also mandatory that a graceful exit of inflation must happen in such a way that the inflaton decay products must excite the standard model (SM) quarks and leptons $[3,4]$ required for the success of big bang nucleosynthesis (BBN) [5]. This can be achieved without any need of ad hoc assumptions provided that the inflaton carries the SM charges as in the case of $[3,4]$.

The above construction is based on embedding inflation within the gauge invariant flat directions of the minimal supersymmetric standard model (MSSM); for a review, see [6,7]. Since the inflaton interactions are that of the SM, the inflaton directly decays into the SM quarks and leptons [3,4], and the lightest supersymmetric particle (LSP) [8]. Note that the dark matter particles are created and matched with the current observations just from their thermal interactions. Moreover, the reheat temperature is sufficiently high enough to create baryon asymmetry before the standard electroweak transitions [6,7].

However, there is also a plethora of models of inflation which do not belong to the observable sector [9]. In such cases the inflaton belongs to the hidden sector whose mass and couplings a priori are not known to us. They can be either an absolute gauge singlet or just a SM gauge singlet, whose couplings to the SM fields are generically considered to be Planck suppressed. In this paper we call them moduli. ${ }^{1}$

The aim of the present paper is to provide a minimal and a successful cosmology prompting from a hidden sector physics which can explain baryogenesis and dark matter at

\footnotetext{
${ }^{1}$ The word moduli is a misnomer here, as it may or may not bear its inkling to that of the string moduli arising from string compactifications [10].
}

ultralow reheat temperatures such as $T_{R} \sim 1-10 \mathrm{MeV}$. Such a stiff challenge can be posed by any inflationary model where the inflaton is a SM gauge singlet.

As we expect there would be many problems which we need to overcome. In any case the lowest mass of such a moduli field is always constrained by the success of BBN. The reheating temperature after the moduli decay into the SM degrees of freedom is represented by

$$
T_{R} \sim \sqrt{\Gamma_{\phi} M_{\mathrm{P}}}=10 \mathrm{MeV}\left(\frac{m_{\phi}}{10^{2} \mathrm{TeV}}\right)^{3 / 2},
$$

where we have used the total decay width of the moduli $\Gamma_{\phi} \sim m_{\phi}^{3} / M_{\mathrm{P}}^{2}$ with the reduced Planck mass $M_{\mathrm{P}} \simeq 2.4 \times$ $10^{18} \mathrm{GeV}$. Since we request $T_{R} \gtrsim 5 \mathrm{MeV}$, in order not to spoil the successes of BBN [11], we have a lower limit on the mass, $m_{\phi} \geq 10^{5} \mathrm{GeV}$. Then, we get a relationship, $T_{R} / m_{\phi} \gtrsim 10^{-7}$.

Challenges for baryonic asymmetry.-If the moduli mass is heavier than $m_{\phi} \geq 10^{7.5}-10^{8} \mathrm{GeV}$, then it is possible to get a reheat temperature above $T_{R} \geq 100 \mathrm{GeV}$. At such reheat temperatures there are many ways to generate matter-antimatter asymmetry such as electroweak baryogenesis, Affleck-Dine baryogenesis, thermal/nonthermal leptogenesis, baryogenesis via Q-ball evaporation, etc. $[6,7]$.

The problem arises when the reheat temperature is of the order of $T_{R} \sim 1-10 \mathrm{MeV}$. For such a low reheating many of the mechanisms for generating matter-antimatter asymmetry will not work. First of all the scale of thermalization and the hadronization ought to be very close to each other [12], such that the Universe could go through a successful phase of BBN. Secondly, one would have to directly create baryons and antibaryons and the tiny asymmetry between them simultaneously. One cannot resort to electroweak sphaleron transitions whose rates are by now exponentially suppressed for $T \ll 100 \mathrm{GeV}$.

In order to create baryon asymmetry we would require all three well-known Sakharov conditions: (1) an out of 
equilibrium scenario, which can be obtained from the decay of the moduli, (2) baryon number violation, and (3) sufficiently large $C P$ asymmetry. The latter issues are challenging from a model building point of view. Within the SM, the $B$ and $L$ are accidental global symmetries; therefore it is not clear that a priori $B$ and $L$ are conserved within the MSSM. As we shall argue here, the only way one can obtain baryon number violation is if one breaks $R$ parity in the hadronic sector in such a way that it is well constrained by the present set of experiments.

The dark matter production.-In order to explain the large scale structures of the Universe, we need to excite the dark matter. However, exciting heavy thermal dark matter, such as a generic LSP mass of order $\sim 100 \mathrm{GeV}$, is a challenging problem at such low reheat temperatures of order $T_{R} \sim 1-10 \mathrm{MeV}$. Typically a thermal freeze-out temperature will be proportional to the LSP mass, $m_{\mathrm{LSP}} / 20 \sim 5-10 \mathrm{GeV}$. Therefore, we would have to create LSP via a nonthermal process from the direct decay of the moduli. An important challenge arises when the $R$ parity is broken; then the LSP would potentially decay into quarks and leptons much before the structures can be formed in the Universe.

$R$-parity violation and baryogenesis. - Given the nature of the issues we are discussing here it is important to understand what are the current limits on $R$-parity violating interactions. Let us now consider a scenario where $B$ and $L$ are violated; then the MSSM superpotential allows the following well-known gauge invariant terms:

$$
\begin{aligned}
W_{R_{b}}= & \mu_{i}^{\prime} L_{i} H_{u}+\lambda_{i j k} L_{i} L_{j} \ell_{k}^{c}+\lambda_{i j k}^{\prime} L_{i} Q_{j} d_{k}^{c} \\
& +\lambda_{i j k}^{\prime \prime} u_{i}^{c} d_{j}^{c} d_{k}^{c},
\end{aligned}
$$

where $L_{i}=\left(\nu_{i}, \ell_{i}\right), Q_{i}=\left(u_{i}, d_{i}\right), H_{u}=\left(h_{u}^{+}, h_{u}^{0}\right)^{T}, H_{d}=$ $\left(h_{d}^{0}, h_{d}^{-}\right)^{T}$, etc. are $S U(2)_{L}$ doublets and $u_{i}^{c}, d_{i}^{c}$ are $S U(2)_{L}$ singlet quarks. In Eq. (2), the first three terms violate the lepton number by one unit $(\Delta L=1)$, while the last term violates the baryon number by one unit $(\Delta B=1)$. For the stability of the proton we assume that $\lambda_{i j k}=\lambda_{i j k}^{\prime}=0$. This can be accomplished if there exists any conservation of lepton number, which then forces $\mu_{i}^{\prime}$ to be zero. Under this condition some of the $\lambda_{i j k}^{\prime \prime}$ couplings are considerably large. However, the nonobservation of certain phenomena gives stringent constraints on these couplings. In particular, the electric dipole moment of neutron gives [13]

$$
\operatorname{Im}\left(\lambda_{312}^{\prime \prime} \lambda_{332}^{\prime \prime}\right)<0.03\left(\frac{0.01}{V_{t d}}\right)\left(\frac{\tilde{M}}{\mathrm{TeV}}\right)^{2} .
$$

Similarly the nonobservation of $n-\bar{n}$ oscillation gives an upper bound on $\lambda_{11 k}^{\prime \prime}$ to be [13]

$$
\left|\lambda_{11 k}^{\prime \prime}\right|<\left(10^{-6}-10^{-5}\right) \frac{10^{8} \mathrm{~s}}{\tau_{\text {osc }}}\left(\frac{\tilde{M}}{\mathrm{TeV}}\right)^{5 / 2} .
$$

Thus we see that $\lambda_{332}^{\prime \prime}$ is hardly constrained and can be taken to be as large as $\mathcal{O}(1)$. We use this to our advantage in order to estimate the baryon asymmetry from the out of equilibrium decay of the moduli.

Let us consider that $\phi$ decays to MSSM degrees of freedom before BBN. Now due to the large branching ratio, the decay of $\phi$ mostly gives rise to gauge bosons and gauginos, although it decays to gravitino, fermion, and sfermions with smaller branching ratios. Since there is a baryon number violation through the $R$-parity violating couplings $\lambda_{i j k}^{\prime \prime}$, the decay of moduli and its decay products, primarily gauginos, will produce a net baryon asymmetry.

First of all note that within the MSSM, the Planck scale suppressed decay of the moduli field, $\phi \rightarrow u_{i} u_{j}^{c}, d_{i} d_{j}^{c}$, does not give rise to a net $C P$ violation up to one loop quantum correction. The $C P$ asymmetry in the moduli decay arises only through the two loop quantum corrections which are suppressed in comparison to the $C P$ asymmetry produced by the decay of gauginos. Therefore, in what follows we will discuss the baryon asymmetry from the decay of gaugino fields (gluino, Z-ino, and photino), represented here as $\tilde{g}$.

Let us assume that the gauginos are heavier than the quarks and squarks. As a result their decay to a pair of quark and squark through one loop quantum correction gives rise to a net $C P$ violation. The magnitude of $C P$ violation in the decay $\tilde{g} \rightarrow t \tilde{t}^{c}$ can be estimated as [14]

$$
\epsilon=\frac{\Gamma\left(\tilde{g} \rightarrow t \tilde{t}^{c}\right)-\Gamma(\tilde{g} \rightarrow \bar{t} \tilde{t})}{\Gamma_{\tilde{g}}^{\mathrm{tot}}} \approx \frac{\lambda_{323}^{\prime \prime}}{16 \pi} \frac{\operatorname{Im}\left(A_{323}^{*} m_{\tilde{g}}\right)}{\left|m_{\tilde{g}}\right|^{2}},
$$

where $A_{323}$ is the trilinear supersymmetric (SUSY) breaking term and we also assume a maximal $C P$ violation. As a result the decay of gauginos produces more squarks (antisqarks) than antisquarks (squarks). The baryon number violating $(\Delta B=1)$ decay, induced by $\lambda_{323}^{\prime \prime}$ of squarks (antisquarks) to quarks (antiquarks) then gives rise to a net baryon asymmetry. Note that the decay of squarks (antisquarks) is much faster than any other processes that would erase the produced baryon asymmetry. Hence the $B$ asymmetry can simply be given by

$$
\eta_{B} \sim B_{\tilde{g}} \epsilon \frac{n_{\phi}}{s} \sim \frac{3}{4} B_{\tilde{g}} \epsilon \frac{T_{R}}{m_{\phi}},
$$

where $B_{\tilde{g}} \sim 0.5$ is the branching ratio of the decay of $\phi$ to $\tilde{g} \tilde{g}$, and in the above equation $s$ is the entropy density resulting through the decay of $\phi$. Let us consider a parameter space set by Eq. (1), where $T_{R} / m_{\phi} \sim 10^{-7}$ and $m_{\phi} \sim 10^{5} \mathrm{GeV}$. Therefore, a reasonable $C P$ violation of order $\epsilon \sim 0.01-0.001$ could accommodate the desired baryon asymmetry of $\mathcal{O}\left(10^{-10}\right)$ close to the temperature of $T \sim 10^{-1} \mathrm{MeV}$.

Gravitino as a dark matter-Let us now consider a possible dark matter candidate in our scenario. Because of violation of $R$ parity, as such the LSP is not completely stable. Therefore, a neutralino-type standard dark matter scenario will not be an able candidate. Because of the large 
$R$-parity violating coupling, either arising from $\lambda_{332}^{\prime \prime}$ or $\lambda_{312}^{\prime \prime}$, the neutralino will decay much before the age of the Universe. The only probable candidate for the dark matter would be the gravitino, whose lifetime will be further suppressed by the Planck suppressed interactions. Furthermore, if the gravitino is the LSP then the two body decay will be prohibited and the only viable channel will be the three body decay into the SM fermions, which will also include the $R$-parity violating coupling, i.e. $\lambda_{323}^{\prime \prime}$.

Let us now consider the gravitino abundance from the moduli decay:

$$
Y_{3 / 2} \sim B_{3 / 2} \frac{3 T_{R}}{4 m_{\phi}}
$$

where $B_{3 / 2}$ is the branching ratio into the gravitino and would be $B_{3 / 2}=10^{-2}-1$ [15] with the mixing between modulus and the supersymmetry-breaking field. We have used an approximation $n_{\phi} / s \sim\left(3 T_{R} / 4 m_{\phi}\right){ }^{2}$

Let us evaluate the gravitino contribution to the density of the dark matter,

$$
Y_{3 / 2}=3 \times 10^{-10}\left(\frac{m_{3 / 2}}{\mathrm{GeV}}\right)^{-1}\left(\frac{\Omega_{3 / 2} h^{2}}{0.11}\right)
$$

where the density parameter of the present Universe is reported by WMAP 5-year to be $\Omega_{\mathrm{CDM}} h^{2} \sim 0.11$ [2] with the normalized Hubble parameter $h$. Note that for a gravitino mass of order $1 \mathrm{GeV}$ we can explain the right dark matter abundance with $B_{3 / 2} \sim 10^{-2}$ and $T_{R} / m_{\phi} \sim$ $10^{-7}$

In the presence of $R$-parity violation it becomes important to ask whether the gravitino can live long enough to serve as a dark matter candidate or not. One can estimate the decay rate of the gravitino induced by the $R$-parity violation, which can be written as

$$
\Gamma_{3 / 2}=\frac{\lambda_{323}^{\prime \prime}{ }^{2}}{192 \pi^{3}} \frac{m_{3 / 2}^{5}}{\tilde{M}^{2} M_{\mathrm{P}}^{2}},
$$

where $\tilde{M}$ is the mass of the supersymmetric particles, i.e. sparticle, which couples to the gravitino and induces threebodies decay. Equation (9) gives the lifetime of the gravitino,

$$
\tau_{3 / 2} \sim 2.3 \times 10^{22} \mathrm{~s}\left(\frac{\lambda_{323}^{\prime \prime}}{0.1}\right)^{-2}\left(\frac{m_{3 / 2}}{\mathrm{GeV}}\right)^{-5}\left(\frac{\tilde{M}}{10^{3} \mathrm{GeV}}\right)^{2} .
$$

Therefore, the lifetime of the gravitino can be longer than

\footnotetext{
${ }^{2}$ The branching ratio of the gravitino production from an absolute gauge singlet is little more contentious than one would expect naively. The moduli decay rate could get a helicity suppression which depends on the details of the SUSY breaking hidden sector [15]. There are examples of hidden sectors, where $B_{3 / 2} \sim 10^{-2}$; see the second and third references in [15].
}

the cosmic age. However, there is an important point to note here. If the gravitino mass is such that $m_{3 / 2} \leq 1 \mathrm{GeV}$, then the gravitino is absolutely stable as there is a kinematical suppression for a gravitino to decay into the SM baryons.

In addition, there is an attractive feature to note here that the gravitino production by the decay of other superparticles is also suppressed and negligible compared with the direct two-bodies decay of the moduli, except for the next LSP (NLSP) SUSY particles. They will be produced by the moduli decay products-either they quickly decay into the NLSP directly or through some cascade decays without producing gravitinos. Because of the $R$-parity violation, which induces the three-bodies decay of NLSP into SM fermions, the lifetime of the NLSP can be much shorter than $10^{-2} \mathrm{~s}$, which evades the strong BBN constraints [16], with its decay width $\Gamma \sim\left(\lambda_{323}^{\prime \prime}\right)^{2} \alpha_{i}^{2} m_{\mathrm{NLSP}}^{3} / \tilde{M}^{2}$, where $\alpha_{i}$ is the fine-structure constant of the gauge coupling and $m_{\mathrm{NLSP}}$ is the NLSP mass. This decay width is much larger than that into the gravitino from the NLSP, which is suppressed by the Planck mass squared. Thus, the production mode of the gravitino is dominated by the decay of moduli into a pair of gravitinos.

For a nonthermal creation of dark matter it is important to check the freestreaming length. The gravitinos can have a large velocity at the radiation matter equality. However, for the parameters we are interested in the freestreaming length comes out to be $\lambda_{\mathrm{FS}} \sim 0.1 \mathrm{Mpc} \log _{e}\left(2 L_{\max }\right)$ $\left(m_{3 / 2} / 1 \mathrm{GeV}\right)^{-1}\left(m_{\phi} / 10^{5} \mathrm{GeV}\right)^{-1 / 2} \quad$ with $\quad L_{\max }=$ $\mathcal{O}\left(10^{2}\right)\left(m_{3 / 2} / 1 \mathrm{GeV}\right)\left(m_{\phi} / 10^{5} \mathrm{GeV}\right)^{1 / 2}$ [17]. For $m_{3 / 2} \sim$ $1 \mathrm{GeV}$, and $m_{\phi} \sim 10^{5} \mathrm{GeV}$, we obtain $\lambda_{\mathrm{FS}} \sim \mathcal{O}(0.1)-$ $1 \mathrm{Mpc}$. Such a freestreaming length is marginal from the point of view of growth in the dark matter fluctuations. The suppression of the density contrast below the freestreaming length results in erasing small structures, which can be tested by comparison between detailed $N$-body simulations and observations of Lyman- $\alpha$ clouds, or future submillilensing observation of subhalos [18].

A model for a hidden sector low scale inflation.-So far we have not discussed the cosmic role of a $\phi$ field. In our case the moduli can act as an inflaton. One can envisage a simple low scale inflationary model where inflation occurs near the point of inflection with a mass $m_{\phi} \sim 10^{5} \mathrm{GeV}$ and a potential:

$$
V(\phi) \sim \frac{m_{\phi}^{2}}{2} \phi^{2}-\frac{A \kappa}{6 \sqrt{3}} \phi^{3}+\frac{\kappa^{2}}{12} \phi^{4}
$$

where $A \approx 4 m_{\phi}$ and $\kappa \sim 10^{-10}$. Inflation can happen near $\phi_{0} \sim \sqrt{3} m_{\phi} / \kappa$ with a Hubble expansion rate, $H_{\mathrm{inf}} \sim$ $\left(m_{\phi}^{2} / \kappa M_{\mathrm{P}}\right) \sim 100 \mathrm{GeV}$. The amplitude of the density perturbations will be given by [4] $\delta_{H} \approx(1 / 5 \pi)\left(H_{\mathrm{inf}}^{2} / \dot{\phi}\right) \sim$ $\left(\kappa^{2} M_{\mathrm{P}} / 3 m_{\phi}\right) \mathcal{N}^{2} \sim 10^{-5}$, where the number of e-foldings is given by $\mathcal{N} \sim 45$. One of the dynamical properties of an 
inflection point inflation is that the spectral tilt can be matched in a desired observable range $0.92<n_{s}<1.0$ for the above parameters; see $[3,19,20]$.

Discussions. - It is also possible to imagine $\phi$ to be a curvaton [21], which dominates the Universe while decaying. It would be desirable to have a curvaton belonging to the observable sector [22], but this need not be the case always. The curvaton model still requires the inflationary potential to dominate the energy density initially, so that the curvaton remains light during inflation. An observed amplitude of perturbations can be created from the decay of the curvaton with a mass of order $10^{5} \mathrm{GeV}$. If the curvaton oscillations dominate, then there will be no distinguishable CMB signatures except the spectral tilt is generically $n_{s} \sim 1$. Since all of radiation, baryon, and dark matter have the same adiabatic perturbations, our model should not suffer from the constraint from isocurvature perturbation (see the discussion in [23]).

Furthermore, one can also imagine obtaining a low scale baryogenesis via the Affleck-Dine mechanism in a $R$-parity violating scenario with a moduli coupling to $u_{i}^{c} d_{j}^{c} d_{k}^{c}$ [24] or $R$-parity violating top decays [25]. The case of Ref. [24] is quite interesting; however, note that in such a scenario the $\phi$ field cannot act as an inflaton. One would require an inflaton sector, and there will be an additional source for baryon isocurvature fluctuations which is already constrained by the current WMAP data [2].

To summarize, we have realized a successful early universe cosmology within a hidden sector inflaton paradigm which gives rise to seed perturbations for the $\mathrm{CMB}$, an observable range of tilt in the power spectrum, and ultralow scale reheat temperatures of order $1-10 \mathrm{MeV}$. The origins of baryogenesis and dark matter in our scenario are now related to the $R$-parity violating interaction of the type $\lambda_{323}^{\prime \prime} u_{3}^{c} d_{2}^{c} d_{3}^{c}$. The baryonic asymmetry is created from the decay products of a singlet inflaton and a viable dark matter candidate is the gravitino. Future experiments such as the electric dipole moment of the neutron, dark matter searches, and the upcoming LHC will be able to constrain our scenario by providing a better handle on $R$-parity violating interactions.

The authors are partly supported by "UNIVERSENET" (No. MRTN-CT-2006-035863) and by STFC Grant No. PP/D000394/1.
[1] C. P. Burgess, R. Easther, A. Mazumdar, D. F. Mota, and T. Multamaki, J. High Energy Phys. 05 (2005) 067; R. Allahverdi, A. R. Frey, and A. Mazumdar, Phys. Rev. D 76, 026001 (2007).

[2] E. Komatsu et al. (WMAP Collaboration), Astrophys. J. Suppl. Ser. 180, 330 (2009).

[3] R. Allahverdi, K. Enqvist, J. Garcia-Bellido, and A. Mazumdar, Phys. Rev. Lett. 97, 191304 (2006); R. Allahverdi, K. Enqvist, J. Garcia-Bellido, A. Jokinen, and A. Mazumdar, J. Cosmol. Astropart. Phys. 06 (2007) 019.

[4] R. Allahverdi, A. Kusenko, and A. Mazumdar, J. Cosmol. Astropart. Phys. 07 (2007) 018.

[5] B. Fields and S. Sarkar, arXiv:astro-ph/0601514.

[6] K. Enqvist and A. Mazumdar, Phys. Rep. 380, 99 (2003).

[7] M. Dine and A. Kusenko, Rev. Mod. Phys. 76, 1 (2003).

[8] R. Allahverdi, B. Dutta, and A. Mazumdar, Phys. Rev. D 75, 075018 (2007); Phys. Rev. Lett. 99, 261301 (2007).

[9] A. D. Linde, Particle Physics and Inflationary Cosmology (Harwood Academic Publishers, Chur, Switzerland, 1990).

[10] J. Polchinski, String Theory. An Introduction to the Bosonic String (Cambridge University Press, Cambridge, UK, 1998), Vol. 1, p. 402.

[11] M. Kawasaki, K. Kohri, and N. Sugiyama, Phys. Rev. Lett. 82, 4168 (1999); Phys. Rev. D 62, 023506 (2000); K. Ichikawa, M. Kawasaki, and F. Takahashi, Phys. Rev. D 72, 043522 (2005).

[12] P. Jaikumar and A. Mazumdar, Nucl. Phys. B683, 264
(2004); T. Asaka, D. Grigoriev, V. Kuzmin, and M. Shaposhnikov, Phys. Rev. Lett. 92, 101303 (2004).

[13] R. Barbier et al., Phys. Rep. 420, 1 (2005).

[14] J. M. Cline and S. Raby, Phys. Rev. D 43, 1781 (1991).

[15] M. Endo, K. Hamaguchi, and F. Takahashi, Phys. Rev. Lett. 96, 211301 (2006); S. Nakamura and M. Yamaguchi, Phys. Lett. B 638, 389 (2006); M. Dine, R. Kitano, A. Morisse, and Y. Shirman, Phys. Rev. D 73, 123518 (2006); M. Endo, K. Hamaguchi, and F. Takahashi, Phys. Rev. D 74, 023531 (2006).

[16] M. Kawasaki, K. Kohri, T. Moroi, and A. Yotsuyanagi, Phys. Rev. D 78, 065011 (2008).

[17] J. Hisano, K. Kohri, and M. M. Nojiri, Phys. Lett. B 505, 169 (2001); M. Kaplinghat, Phys. Rev. D 72, 063510 (2005); J. A. R. Cembranos, J. L. Feng, A. Rajaraman, and F. Takayama, Phys. Rev. Lett. 95, 181301 (2005); F. Takahashi, Phys. Lett. B 660, 100 (2008).

[18] J. Hisano, K. T. Inoue, and T. Takahashi, Phys. Lett. B 643, 141 (2006).

[19] R. Allahverdi, B. Dutta, and A. Mazumdar, Phys. Rev. D 78, 063507 (2008).

[20] J. C. Bueno Sanchez, K. Dimopoulos, and D. H. Lyth, J. Cosmol. Astropart. Phys. 01 (2007) 015.

[21] K. Enqvist and M. S. Sloth, Nucl. Phys. B626, 395 (2002); D. H. Lyth and D. Wands, Phys. Lett. B 524, 5 (2002); T. Moroi and T. Takahashi, Phys. Lett. B 522, 215 (2001); 539, 303(E) (2002); D. H. Lyth, C. Ungarelli, and D. Wands, Phys. Rev. D 67, 023503 (2003).

[22] K. Enqvist, S. Kasuya, and A. Mazumdar, Phys. Rev. Lett. 
90, 091302 (2003); K. Enqvist, A. Jokinen, S. Kasuya, and A. Mazumdar, Phys. Rev. D 68, 103507 (2003); K. Enqvist, S. Kasuya, and A. Mazumdar, Phys. Rev. Lett. 93, 061301 (2004); R. Allahverdi, K. Enqvist, A. Jokinen, and A. Mazumdar, J. Cosmol. Astropart. Phys. 10 (2006) 007.
[23] M. Lemoine, J. Martin, and J. Yokoyama, arXiv:0904.0126.

[24] R. Kitano, H. Murayama, and M. Ratz, Phys. Lett. B 669, 145 (2008).

[25] A. D. Dolgov and F. R. Urban, Nucl. Phys. B752, 297 (2006). 